\begin{tabular}{ccc|} 
ISSN = 1980-993X - doi:10.4136/1980-993X \\
www.ambi-agua.net \\
E-mail: ambi-agua@agro.unitau.br \\
Tel.: (12) 3625-4212
\end{tabular}

\title{
Especies vegetales y síndromes de dispersión del área de protección ambiental municipal de Inhamum, Caxias, Maranhão, Brasil
}

\author{
(http://dx.doi.org/10.4136/ambi-agua.190)
}

\section{Gonçalo Mendes da Conceição ; Ana Cláudia Ruggieri² ${ }^{2}$ Eduardo Oliveira Silva $^{3}$; Ellismary Castelo Gomes ${ }^{4}$; Hilda Margarita Valdesprieto Roche ${ }^{5}$}

\footnotetext{
${ }^{1}$ Doutorando do Programa de Pós-Graduação em Zootecnia - UNESP/Jaboticabal; Universidade Estadual do Maranhão/Centro de Estudos Superiores de Caxias - CESC/UEMA; Núcleo de Pesquisa dos Recursos Biológicos dos Cerrados Maranhenses/RBCEM; e-mail: hyophila@yahoo.com.br,

${ }^{2,1}$ Programa de Pós-Graduação em Zootecnia - UNESP/Jaboticabal; e-mail: acruggieri@fcav.unesp.br, ${ }^{2}$ Mestrando do Programa de Pós-Graduação em Ciências Biológicas - Botânica Tropical, Universidade Federal Rural da Amazônia/Museu Paraense Emílio Goeldi; e-mail: eoliveira12@yahoo.com.br,

${ }^{4}$ Acadêmica do Curso de Ciências/Biologia, da Universidade Estadual do Maranhão/Centro de Estudos Superiores de Caxias - CESC/UEMA; e-mail: lilic.x@hotmail.com,

${ }^{5}$ Doutora, Professora do Curso de Ciências/Biologia, da Universidade Estadual do Maranhão/Centro de Estudos Superiores de Caxias - CESC/UEMA; e-mail: mvaldesprieto@gmail.com,
}

\section{RESUMEN}

El objetivo de este trabajo fue determinar los síndromes de dispersión de las especies vegetales encontradas en el Área de Protección Ambiental Municipal de Inhamum. Para el levantamiento de datos, se utilizó el método de cuadrantes en tres áreas de sabana, localizándose 42 puntos en cada área con inter distanciamiento de $10 \mathrm{~m}$. Fueron muestreados 476 individuos, representados por 19 familias, 33 géneros y 33 especies. Las familias con mayor representatividad de especies fueron Anacardiaceae, Caesalpiniaceae, Fabaceae y Mimosaceae, cada una con tres especies. En cuanto al número de individuos, se destacaron las familias Fabaceae (157 individuos) y Mimosaceae (111 individuos). La especie con mayor número de individuos fue Bowdichia virgilloides (119 individuos). En cuanto a los síndromes de dispersión de las especies en estudio, fue verificado que 271 son anemocóricos (57\%), 117 individuos $(24,5 \%)$ son zoocóricos, y 88 autocóricos $(18,5 \%)$. Al tratar cada área separadamente, la sabana sensu stricto obtuvo 24 especies, siendo ocho $(33,3 \%)$ zoocóricas, $13(54,2 \%)$ anemocóricas y tres (12,5\%) autocóricas. Para la sabana fueron encontradas 26 especies, en que $11(42,3 \%)$ zoocóricas, $12(46,2 \%)$ anemocóricas y tres $(11,5 \%)$ autocóricas. Ya para el campo sucio, fueron obtenidas 28 especies, siendo $13(46,4 \%)$ zoocóricas, 12 $(42,9 \%)$ anemocóricas y tres $(10,7 \%)$ autocóricas. El síndrome anemocórica, por ser típica de la sabana fue el mejor representado en las tres áreas. Se infiere mediante los resultados presentados que el alto índice de especies anemocóricas sea en consecuencia del nivel de antropización de la sabana del área estudiada.

Palabras-clave: Diáspora; dispersión; conservación; ecología de comunidad. 


\title{
Plant species and dispersal syndromes from the environmental protection areas of the city of Inhamum, Caxias, Maranhão, Brazil
}

\begin{abstract}
The present study was aimed to determine the dispersal syndromes of plant species found in the area for environmental protection of Inhamum. Data collection were performed using the quadrant method in three areas of the Cerrado, with allocation of 42 points in each area with a 10 meter spacing. The sample was composed of 476 individuals represented by 19 families, 33 genera and 33 species. The families with the greatest representativeness were: Anacardiaceae, Caesalpiniaceae, Fabaceae and Mimosaceae, each one of them with three species. The most representative families based on the number of individuals were: Fabaceae (157 individuals) and Mimosaceae (111 individuals). The species with the largest number of individuals was Bowdichia virgilloides (119 individuals). Considering the dispersal syndromes of the study species, it has been found that 271 were anemochorous (57\%), 117 individuals $(24.5 \%)$ zoochorous, and 88 autochoric $(18.5 \%)$. Considering each area was separately, the Cerrado stricto sensu had 24 species, with eight (33.3\%) being zoochorous, 13 (54.2\%) anemochorous and three (12.5\%) autochoric. In the so-called Cerradão, 26 species were found among them, $11(42.3 \%)$ were zoochorous, $12(46.2 \%)$ anemochorous and three $(11.5 \%)$ autochorous. In the Campo sujo, 28 species were found, with $13(46.4 \%)$ zoochorous, $12(42.9 \%)$ anemochorous and three (10.7\%) autochoric. The anemochorous species is typical of the Cerrados and, therefore, it was the best represented species in the three areas. Based upon these findings it can be inferred that the large number of anemochorous species is due to the level of anthropization of the Cerrado in the study area.
\end{abstract}

Keywords: Diaspore; dispersal; preservation; community ecology.

\section{Espécies vegetais e síndromes de dispersão, da área de proteção ambiental municipal do Inhamum, Caxias, Maranhão, Brasil}

\section{RESUMO}

O objetivo do trabalho foi determinar as síndromes de dispersão das espécies vegetais encontradas na Área de Proteção Ambiental Municipal do Inhamum. Para o levantamento de dados, utilizou-se o método de quadrantes em três áreas de cerrado, alocando-se 42 pontos em cada área com interdistanciamento de $10 \mathrm{~m}$. Foram amostrados 476 indivíduos, representados por 19 famílias, 33 gêneros e 33 espécies. As famílias com maior representatividade de espécies foram: Anacardiaceae, Caesalpiniaceae, Fabaceae e Mimosaceae, cada uma com três espécies. Quanto ao número de indivíduos, destacaram-se as famílias Fabaceae (157 indivíduos) e Mimosaceae (111 indivíduos). A espécie com maior número de indivíduos foi Bowdichia virgilloides (119 indivíduos). Quanto às síndromes de dispersão das espécies em estudo, foi verificado que 271 são anemocóricos (57\%), 117 indivíduos (24,5\%) zoocóricos, e 88 autocóricos $(18,5 \%)$. Ao tratar cada área separadamente, o Cerrado sensu stricto obteve 24 espécies, sendo oito $(33,3 \%)$ zoocóricas, $13(54,2 \%)$ anemocóricas e três $(12,5 \%)$ autocóricas. Para o Cerradão foram encontradas 26 espécies, em que 11 (42,3\%) são zoocóricas, 12 (46,2\%) anemocóricas e três $(11,5 \%)$ autocóricas. Já para o Campo sujo, foram obtidas 28 espécies, sendo $13(46,4 \%)$ zoocóricas, 12 (42,9\%) anemocóricas e três (10,7\%) autocóricas. A síndrome anemocórica, por ser típica dos cerrados, foi a mais bem representada nas três áreas. Infere-se diante dos resultados apresentados que o alto índice de espécies anemocóricas seja em decorrência do nível de antropização do cerrado da área estudada.

Palavras-chave: Diásporo; dispersão; conservação; ecologia de comunidade. 


\section{INTRODUCCIÓN}

Brasil posee una extensión territorial de 854.740.300 hectáreas, sometidas a una variedad de condiciones climáticas que permiten el desarrollo de una gran diversidad de ambientes (Mesquita y Castro, 2007). Entre estos, la sabana es el segundo mayor bioma brasileño, siendo superado en área apenas por la Amazonia. Ocupa 21\% del territorio nacional y es considerado la última frontera agrícola del planeta. El término sabana es comúnmente utilizado para designar el conjunto de ecosistemas (sabanas, selvas, campos y selvas de galería) que se encuentran en Brasil Central (Klink y Machado, 2005). Este se caracteriza por presentar dos períodos climáticos: uno seco y otro lluvioso, presentando diversos tipos de formaciones herbáceas, arbustivas y arbóreas (Ribeiro y Walter, 1998).

La sabana se extiende por un área continua de Brasil Central y se encuentra también en áreas discontinuas al Norte y al Sur del País, siendo caracterizado por una gran variación de tipos fisonómicos de vegetación, representados por formaciones forestales, de sabana y campestres (Martins et al., 2007), siendo este reconocido como el nacimiento de las aguas, por abrigar importantes cuencas hidrográficas de la América del Sur (Rebelo et al., 2009).

Algunos estudios florísticos y fitosociológicos, sobre todo del componente arbóreo y arbustivo, ya fueron realizados, no sólo en las áreas centrales sino también en las áreas distantes de las sabanas. Esos trabajos mostraron que la vegetación de las sabanas presenta fisionomías típicas, elevada diversidad florística y especies con amplia distribución geográfica (Costa y Araújo, 2000).

Una de las mayores concentraciones de las sabanas en el Nordeste del Brasil se encuentra en los estados de Piauí y de Maranhão, ocupando las porciones Sudoeste y Centro - Norte (Piauí) y Centro-Sur y Nordeste (Maranhão), cubriendo un área estimada de 21.656.866 ha, lo que corresponde a cerca de $14 \%$ del área total de la Región Nordeste, o sea, cerca de 10,8\% del área total de las sabanas sensu lato brasileña (Castro et al., 2007).

Maranhão tiene una posición de transición entre las regiones Norte, Nordeste y CentroOeste. Se localiza entre las coordenadas geográficas $01^{\circ} 01^{\prime} 00^{\prime \prime} / 10^{\circ} 21^{\prime} 07^{\prime \prime} \mathrm{S}$ y 41 $48^{\prime} 30^{\prime} / 48^{\circ} 40^{\prime} 5^{\prime}$ 'W, con una extensión territorial de 332, 174 km² (Maranhão, 1991). El Estado posee cerca de aproximadamente 10.000 .000 ha de sabana, o sea, 30\% de la extensión territorial y 5\% del área total de sabana brasileña. Fisiográficamente el estado de Maranhão está dividido en siete regiones: litoral, depresión, sabanas, coqueros, pre-amazónica, mesetas y altiplanicies. El área de sabana sensu lato en el estado se encuentra localizada en casi todas las regiones fisiográficas, principalmente, en las sabanas, planicies, mesetas y altiplanicies . Se trata de una región con suelos de baja fertilidad, elevada acidez, poseyendo en su gran mayoría, poca agua disponible para las plantas, mas, predominantemente mecanizables para la agricultura (Conceição y Castro, 2009).

Un aspecto importante en el estudio de la ecologia de la sabana se refiere a la caracterización de los patrones de dispersión de diásporas en sus fitofisionomías. La dispersión es definida como la salida o retirada de la diáspora de la planta madre y presenta como ventaja evitar la mortalidad de semillas y plántulas próximas a la planta madre y la ocupación de locales favorables a la germinación y sobre vivencia de las especies dispersadas, constituyendo-se en un proceso de vital importancia para el suceso reproductivo de las especies en la comunidad vegetal (Martins et al., 2007; Trindade et al., 2007). Siendo así, la reproducción de plantas tropicales depende, esencialmente, de la interacción con animales polinizadores y/o dispersores de semillas, una vez que las angiospermas necesitan de animales para el transporte de polen o de sus semillas (Stefanello et al., 2010). Por tanto, uno de los factores que influyen en la colonización de hábitats, es el mecanismo de dispersión de las semillas (Van Der Pijl, 1982).

La dispersión también modifica los patrones de reclutamiento en escala espacial y temporal. Para especies dependientes de condiciones especiales para el establecimiento de 
plántulas, con espacios vacíos, con ocurrencia espacial y temporal variable en el ambiente forestal, el transporte de diásporas es esencial para la colonización de nuevos sitios. De esa forma, la estructura y la dinámica de las comunidades vegetales son influenciadas por la dinámica de la dispersión (Giehl et al., 2007).

La dispersión de semillas, también es importante para la recuperación de áreas degradadas por actividades antrópicas, pues, para que se obtengan paisajes armoniosos y auto-sustentables, es necesario que se considere la sucesión vegetal en la recuperación de esas áreas (Liebsh y Acra, 2007; Yamamoto et al., 2007).

Con base en los criterios morfológicos descritos por Van der Pijl (1982), los frutos son clasificados en tres grandes grupos en cuanto a su modo de dispersión: anemocóricos, cuando las diásporas son adaptadas a la dispersión por el viento; zoocóricos, en que las diásporas son adaptadas a la dispersión por animales y autocóricos, en que las diásporas son dispersas por gravedad o dehiscencia explosiva. Entre tanto, la frecuencia de estas estrategias de dispersión de semillas, difiere entre locales húmidos y secos, así como entre especies arbóreas, perennes y decíduas (Bullock, 1995; Gentry, 1995). Generalmente las semillas dispersas por el viento prevalecen en lugares de flora seca y la dispersión por animales gana más importancia en florestas húmedas (Howe y Smallwood, 1982).

Las diferentes maneras con que las diásporas son dispersas y la frecuencia con que alcanzan ambientes favorables para el establecimiento de la plántula es que determinan la riqueza y la distribución espacial de las poblaciones de plantas (Van Der Pijl, 1982).

En la composición florística de la mayoría de las florestas tropicales y subtropicales existe mayor proporción de especies arbóreas con diásporas propensas a dispersión zoocórica, seguidas por anemocóricas y autocóricas (Giehl et al., 2007).

Dentro de esta temática, varios son los autores que tratan de la dispersión de semillas en áreas de sabana: (Trindade et al., 2007; Seleme et al., 2009; Stefanello et al., 2009; Luz et al., 2008; Martins et al., 2007; Vieira et al., 2002).

Considerando el contexto expuesto, este trabajo tuvo como objetivo realizar el levantamiento florístico y la caracterización de los síndromes de dispersión de diásporas de las especies arbustivo-arbóreas de tres fitofisionomias da sabana del Área de Protección Ambiental Municipal de Inhamum (Caxias/Maranhão), Brasil.

\section{MATERIALES Y MÉTODOS}

\subsection{Localización y caracterización del área de estudio}

El municipio de Caxias/MA, pertenece a la zona fisiográfica del rio Itapecuru, situado en la meso región del este maranhense, con área de 5.313,2 $\mathrm{km}^{2}$, limitado al Norte por los municipios de Codó, Aldeias Altas y Coelho Neto, al Sur, por los municipios de São João do Sóter, Parnarama, Matões y Timon, al este por el estado de Piauí y al Oeste por el municipio de Codó, presentando las siguientes coordenadas geográficas: Latitud 0451'30' Sur y Longitud de $43^{\circ} 21^{\prime} 22^{\prime}$ ' a Oeste, y altitud en torno de 66 metros (Conceição et al., 2010).

El clima es de tipo sub-húmedo seco, con temperatura anual en torno de $27^{\circ} \mathrm{C}$, humedad relativa del aire entre $70 \%$ a $73 \%$, precipitación pluviométrica entre 1600 a $2000 \mathrm{~mm}$, con dos estaciones bien definidas, una lluviosa de diciembre a junio y una seca de julio a noviembre. Los suelos predominantes son lacto suelo rojizo-amarillo, podzólico rojizo-amarillo, arenas y suelos aluviales. La fitofisionomía predominante en el municipio es la flora estacional semidesidual, con predominio de "babaçuais", en algunos trechos el palmera "babaçu (Orbignya pharelata Mart.)" deja de existir y en esos trechos se evidencia sabana y bosque (Reis y Conceição, 2010).

El Área de Protección Ambiental Municipal de Inhamum (Figura 1) está localizada entre las coordenadas $04^{\circ} 53^{\prime} 30^{\prime}$ 'S $/ 43^{\circ} 24^{\prime} 53^{\prime} \mathrm{W}$, a la margen izquierda de la estrada BR-316, siendo cortada verticalmente por la MA-127 que conecta Caxias a São João do Sóter/MA, poseyendo 
un área de aproximadamente 4.500 ha. Se caracteriza por presentar una vegetación típica de sabana, que va desde sabana clara hasta bosque denso. A lo largo de las ramblas, lagunas y nascientes, la vegetación es formada por selva ciliar o selva de galeria (Conceição et al., 2010)

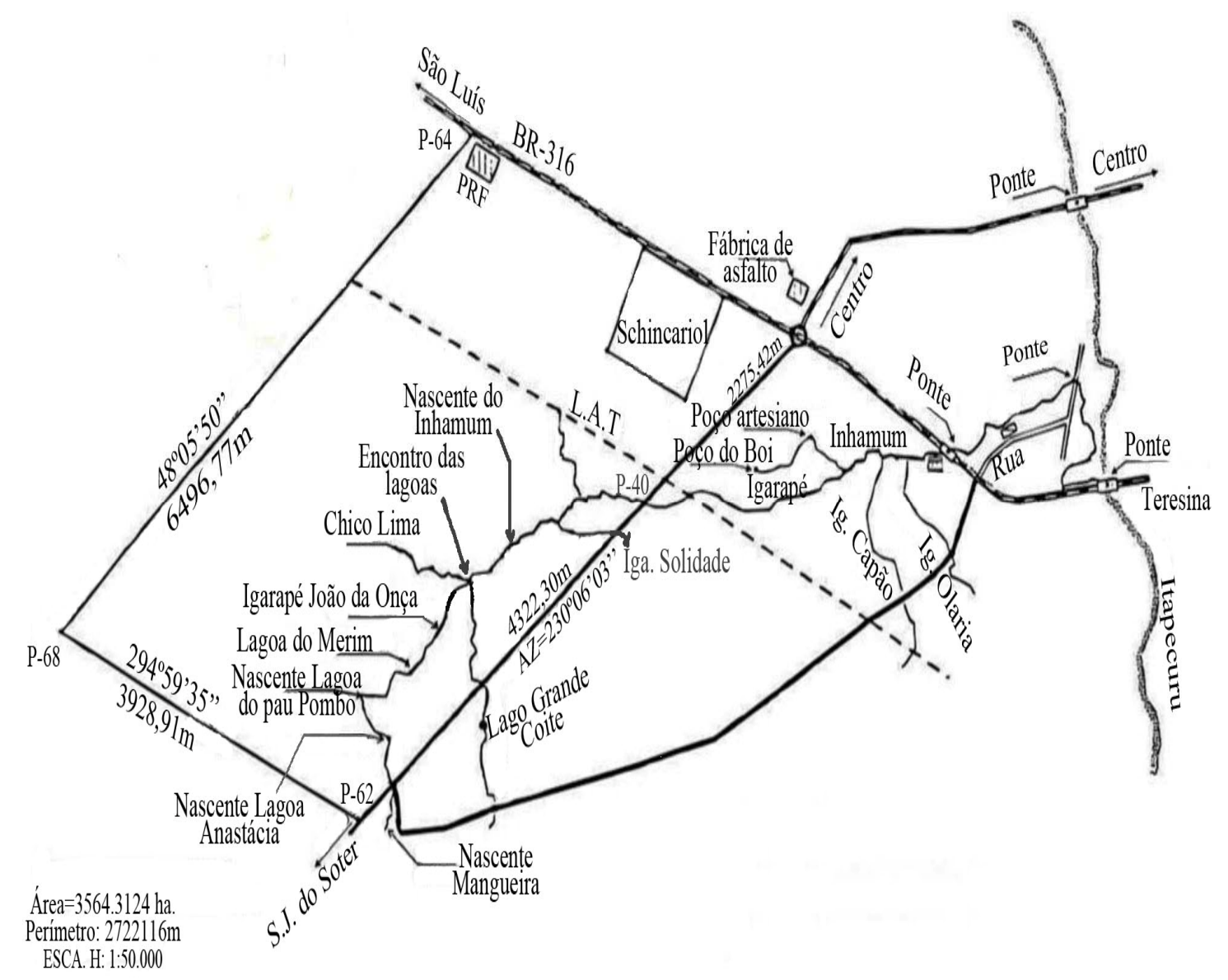

Figura 1. Mapa del Área de Protección Ambiental Municipal del Inhamum, evidenciando su red de drenaje (Caxias, 2001).

\subsection{Levantamiento florístico de las especies estudiada}

Para el levantamiento florístico, fueron muestreados todos los individuos vivos seleccionados a través del método de cuadrantes. En fichas de campo, fueron anotados datos referentes como nombre vulgar, perímetro y altura. Las alturas totales fueron determinadas con el uso de una asta telescópica regulable, graduada a cada $0,5 \mathrm{~m}$, conformando un total de $4 \mathrm{~m}$ con el asta toda distendida. Individuos mayores que $4 \mathrm{~m}$ tuvieron sus alturas estimadas visualmente a partir de esa altura.

Las especies fueron identificadas por bibliografia especializada y comparación con ejemplares de muestra existentes en el Herbario Prof. Aluízio Bittencourt (HERBIT), del Centro de Estudios Superiores de Caxias, de la Universidad Estatal de Maranhão/UEMA.

\subsection{Técnica de muestreo}

Dentro del Área de Protección Ambiental Municipal de Inhamum, fueron muestreadas tres fitofisionomias distintas de vegetación (sensu stricto, cerradão y campo sucio).

En cada área fueron localizados 42 puntos cuadrantes, distribuidos a lo largo de siete líneas paralelas, seis puntos de cuadrantes en cada línea, con cuatro individuos muestreados en cada punto. Los puntos fueron representados por estacas con inter distanciamiento de $10 \mathrm{~m}$, de modo que un mismo árbol no fuera muestreado por puntos distintos (Martins y Santos, 
1999). En cada punto, fueron considerados los cuatro individuos vivos más próximos, con diámetro del tallo al nivel del suelo (DNS) igual o superior a $3 \mathrm{~cm}$ y altura mínima de $1 \mathrm{~m}$.

Todas las especies muestreadas fueron clasificadas según los criterios de Van der Pijl (1982) como anemocóricas (dispersas por el viento), zoocóricas (dispersas por animales) y autocóricas (auto-dispersión). La identificación de las síndromes de dispersión fue hecha por medio de consultas a la literatura especializada.

\section{RESULTADOS Y DISCUSIÓN}

En las tres áreas de sabana, fueron muestreados 476 individuos distribuidos en 19 familias, 33 géneros y 33 especies (Tabla 1).

Tabla 1. Lista de familias, especies, nombre vulgar (NV), número de individuos (NI), síndromes de dispersión (SD) (Zoo: zoocórica; Ane: anemocórica; Aut: autocórica) e áreas de ocurrencia (A- Sabana sensu stricto, B- Bosque y C- campo sucio) de plantas encontradas en el Área de Protección Ambiental Municipal de Inhamum (Caxias/Maranhão).

\begin{tabular}{|c|c|c|c|c|}
\hline FAMILIA/ESPECIE & NV & NI & SD & $\begin{array}{l}\text { OCORRÊNC } \\
\text { IA }\end{array}$ \\
\hline Anacardiaceae & & & & \\
\hline Anacardium occidentale $\mathrm{L}$. & Caju & 10 & Zoo & $\mathrm{A}, \mathrm{B}, \mathrm{C}$ \\
\hline Astronium fraxinifolium Schott ex Spreng. & Gonçalo-alves & 06 & Ane & A, B \\
\hline Myracrodruon urundeuva Allem. & Aroeira & 03 & Ane & $\mathrm{A}, \mathrm{B}, \mathrm{C}$ \\
\hline Annonaceae & & & & \\
\hline Annona dioica A.St.-Hil. & Bruto & 05 & Zoo & $\mathrm{B}, \mathrm{C}$ \\
\hline Apocynaceae & & & & \\
\hline Himatanthus obovatus (Müll. Arg.) Woodson & Pau-de-leite & 10 & Ane & $\mathrm{A}, \mathrm{B}, \mathrm{C}$ \\
\hline Aspidosperma spruceanum Benth. ex Müll. Arg. & Pítia & 20 & Ane & $\mathrm{A}, \mathrm{B}$ \\
\hline Hancornia speciosa Gomes & Mangaba & 01 & Ane & A \\
\hline Bignoniaceae & & & & \\
\hline Zeyhera montana Mart. & Bolsa-de-pastor & 09 & Ane & $\mathrm{A}, \mathrm{B}, \mathrm{C}$ \\
\hline $\begin{array}{l}\text { Tabebuia impetiginosa (Mart. ex DC.) Standl. } \\
\text { Buseraceae }\end{array}$ & Pau-d'arco-roxo & 04 & Ane & A, C \\
\hline $\begin{array}{l}\text { Protium heptaphyllum (Aubl.) March. } \\
\text { Caesalpiniaceae }\end{array}$ & Amescla & 01 & Zoo & $\mathrm{C}$ \\
\hline Hymenaea stigonocarpa Mart. ex Hayne & Jatobá & 11 & Zoo & $\mathrm{A}, \mathrm{B}, \mathrm{C}$ \\
\hline Copaifera langsdorffii Desf. & Podói & 09 & Zoo & $\mathrm{B}, \mathrm{C}$ \\
\hline $\begin{array}{l}\text { Dimorphandra gardneriana Tul. } \\
\text { Caryocaraceae }\end{array}$ & Fava-d'anta & 19 & Aut & $\mathrm{A}, \mathrm{B}, \mathrm{C}$ \\
\hline $\begin{array}{l}\text { Caryocar coriaceum Wittm. } \\
\text { Chrysobalanaceae }\end{array}$ & Pequi & 03 & Zoo & $\mathrm{A}, \mathrm{B}, \mathrm{C}$ \\
\hline $\begin{array}{l}\text { Exellodendron cordatum (Hook.f.) Prance } \\
\text { Combretaceae }\end{array}$ & Pau-pombo & 01 & Zoo & $\mathrm{C}$ \\
\hline Combretum mellifluum Eichler & Mufumbo & 04 & Ane & $\mathrm{A}, \mathrm{B}, \mathrm{C}$ \\
\hline $\begin{array}{l}\text { Terminalia brasilienses (Cambess. ex A.St.-Hil.) } \\
\text { Eichler }\end{array}$ & Chapada & 08 & Ane & B \\
\hline
\end{tabular}




\begin{tabular}{|c|c|c|c|c|}
\hline $\begin{array}{l}\text { Ebenaceae } \\
\text { Diospyros hispida A.DC. }\end{array}$ & Olho-de-boi & 03 & Zoo & $\mathrm{A}, \mathrm{C}$ \\
\hline Euphorbiaceae & & & & \\
\hline $\begin{array}{l}\text { Manihot maracasensis Ule } \\
\text { Fabaceae }\end{array}$ & maniçoba & 26 & Aut & $\mathrm{A}, \mathrm{B}, \mathrm{C}$ \\
\hline Bowdichia virgilioides Kunth & $\begin{array}{l}\text { sucupira- } \\
\text { amarela }\end{array}$ & 119 & Ane & $\mathrm{A}, \mathrm{B}, \mathrm{C}$ \\
\hline Pterodon emarginatus Vogel & sucupira-preta & 15 & Ane & $\mathrm{A}, \mathrm{B}, \mathrm{C}$ \\
\hline Vatairea macrocarpa (Benth.) Ducke & amargoso & 23 & Ane & $\mathrm{A}, \mathrm{B}, \mathrm{C}$ \\
\hline Malpighiaceae & & & & \\
\hline $\begin{array}{l}\text { Byrsonima crassifolia (L.) Kunth } \\
\text { Melastomataceae }\end{array}$ & Murici & 26 & Zoo & $\mathrm{A}, \mathrm{B}, \mathrm{C}$ \\
\hline $\begin{array}{l}\text { Mouriri pusa Gardner ex Gardner } \\
\text { Mimosaceae }\end{array}$ & Puçá & 03 & Zoo & $\mathrm{A}, \mathrm{B}$ \\
\hline Parkia platycephala Benth. & $\begin{array}{l}\text { Faveira-de- } \\
\text { bolota }\end{array}$ & 43 & Aut & $\mathrm{A}, \mathrm{B}, \mathrm{C}$ \\
\hline Stryphnodendron coriaceum Benth. & Barbatimão & 33 & Zoo & $\mathrm{A}, \mathrm{B}, \mathrm{C}$ \\
\hline $\begin{array}{l}\text { Plathymenia reticulata Benth. } \\
\text { Myrtaceae }\end{array}$ & Candeia & 35 & Ane & $\mathrm{A}, \mathrm{B}, \mathrm{C}$ \\
\hline $\begin{array}{l}\text { Pisidium hians DC. } \\
\text { Opiliaceae }\end{array}$ & Araçá & 02 & Zoo & $\mathrm{B}, \mathrm{C}$ \\
\hline $\begin{array}{l}\text { Agonandra brasilienses Miers } \\
\text { Rubiaceae }\end{array}$ & Pau-marfim & 01 & Zoo & $\mathrm{C}$ \\
\hline Tocoyena formosa (Cham. \& Schltdl.) K.Schum. & Jenipapo & 08 & Zoo & $\mathrm{A}, \mathrm{B}, \mathrm{C}$ \\
\hline $\begin{array}{l}\text { Alibertia edulis (Rich.) A.Rich.ex.DC. } \\
\text { Vochysiaceae }\end{array}$ & Maria-pretinha & 01 & Zoo & B \\
\hline Qualea parviflora Mart. & Pau-terrinha & 03 & Ane & $\mathrm{B}, \mathrm{C}$ \\
\hline Salvertia convallariodora A.St.-Hil. & Folha-larga & 11 & Ane & $\mathrm{A}, \mathrm{B}, \mathrm{C}$ \\
\hline
\end{tabular}

Las familias más representativas al nivel de especie fueron Anacardiaceae, Apocynaceae, Caesalpiniaceae, Fabaceae y Mimosaceae, cada una con tres especies. En cuanto al número de individuos, se destacaron las familias Fabaceae (157 individuos) y Mimosaceae (111 individuos). Ya la especie con mayor representatividad en el área, fue Bowdichia virgilioides H.B.\&K. (119 individuos).

De las especies muestreadas, 15 (45,5\%) son zoocóricas, 15 (45,5\%) anemocóricas y tres (9\%) autocóricas (Figura 2). Para el total de individuos, 117 (24,5\%) son zoocóricos, 271 (57\%) anemocóricos y $88(18,5 \%)$ autocóricos (Figura 3). Específicamente, no hubo variación de proporción entre anemocoria y zoocoria. Ya en relación al número de individuos, esos porcentuales son bien distintos.

La familia Mimosaceae presentó especies con dispersión autocórica (Parkia platycephala Benth.), zoocórica (Stryphnodendron coriaceum Benth.) y anemocórica (Plathymenia reticulata Benth.). Las familias Apocynaceae $y$ Fabaceae fueron representadas exclusivamente por especies anemocóricas. 


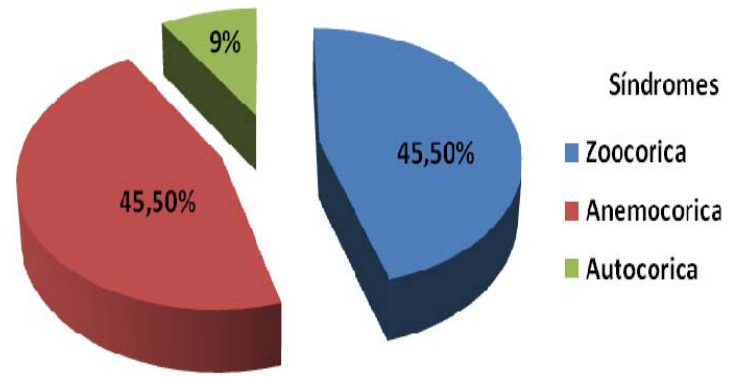

Figura 2. Porcentaje de la distribución de los síndromes de dispersión en las especies muestreadas en el Área de Protección Ambiental Municipal de Inhamum (Caxias/Maranhão).

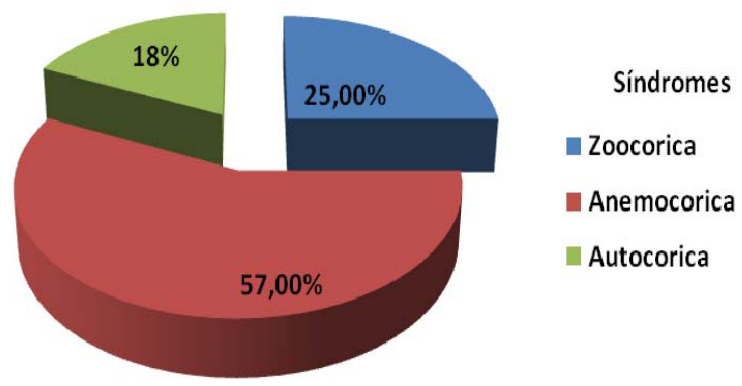

Figura 3. Porcentaje de distribución de síndromes por el total de individuos muestreados, del Área de Protección Ambiental Municipal del Inhamum (Caxias/Maranhão).

Al tratar cada área separadamente, la sabana sensu stricto registró 24 especies, siendo 8 (33,3\%) zoocóricas, 13 (54,2\%) anemocóricas y 3 (12,5\%) autocóricas. Para el bosque fueron encontradas 26 especies, en que $11(42,3 \%)$ son zoocóricas, $12(46,2 \%)$ anemocóricas y 3 $(11,5 \%)$ autocóricas. Ya para el campo sucio, fueron registradas 28 especies, siendo 13 (46,4\%) zoocóricas, 12 (42,9\%) anemocóricas y 3 (10,7\%) autocóricas (Figura 4).

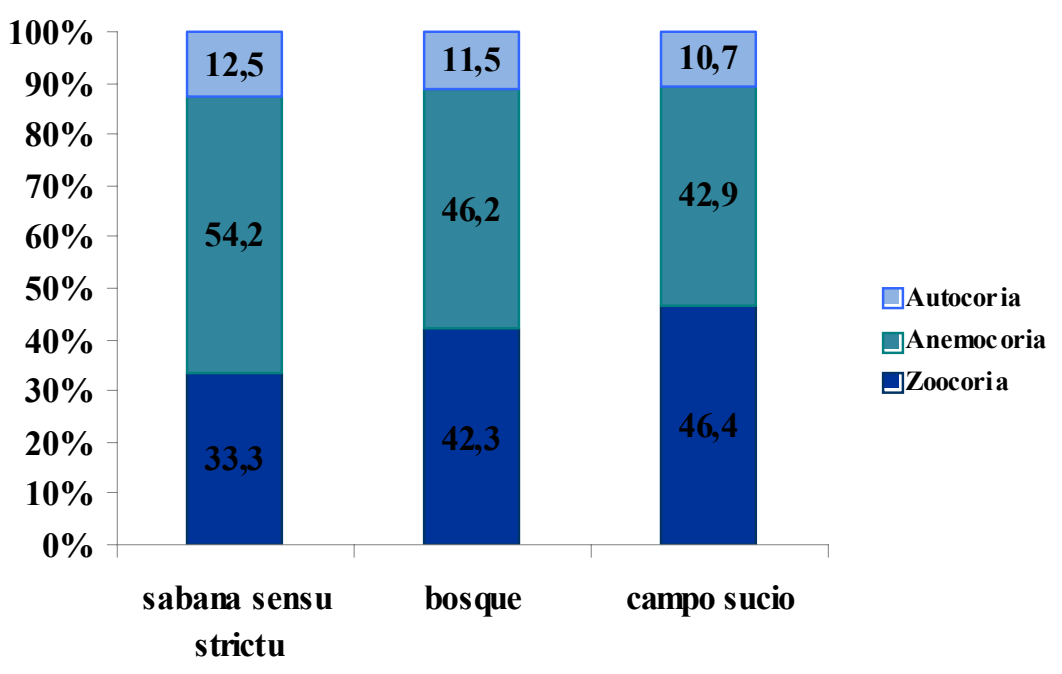

Figura 4. Comparación de la distribución de los síndromes de dispersión por especies muestreadas en las tres áreas de sabana del Área de Protección Ambiental Municipal del Inhamum (Caxias/Maranhão).

Todos los individuos fueron distribuidos en tres clases de altura $(h)$ : pequeños $(h \leq 2,0)$, medios $(2,0<\mathrm{h} \leq 4,0)$ y grandes $(\mathrm{h}>4)$. Hubo una dominancia numérica de especies de 
vegetación, dentro de la clase de altura mayor que 4,0 m (236 individuos) como se puede observar en la Tabla 2. La primera clase fue representada por Alibertia edulis (Rich.) A. Rich. ex. DC. (zoocoria) y Agonandra brasiliensis Miers. (zoocórica). En la segunda clase, se registran especies como Manihot maracasensis Ule. (autocórica) y Tocoyena formosa K. Schum. (zoocórica), entre otras. Con frecuencia mayor, la tercera clase fue representada por varias especies como Caryocar coriaceum Wittm. (zoocórica), Bowdichia virgilioides H.B.\&K. (anemocórica) y Pterodon emarginatus Vogel. (anemocórica). La anemocoria fue predominante en todas las clases de altura (Tabla 3 ).

Tabla 2. Lista de datos numéricos de las especies muestreadas (número de individuos y altura), en el Área de Protección Ambiental Municipal de Inhamum (Caxias/Maranhão).

\begin{tabular}{|c|c|c|}
\hline Nome científico & $\mathbf{N}^{0}$ ind. & Altura Máx. (m) \\
\hline Anacardium occidentale L. & 10 & 7,0 \\
\hline Astronium fraxinifolium Schott ex Spreng. & 6 & 3,0 \\
\hline Myracrodruon urundeuva Allem. & 3 & 6,0 \\
\hline Himatanthus obovatus (Müll.Arg.) Woodson & 10 & 12,0 \\
\hline Aspidosperma spruceanum Benth.ex.Müll.Arg. & 20 & 7,0 \\
\hline Hancornia speciosa Gomes & 1 & 4,3 \\
\hline Annona dioica A.St.-Hil. & 5 & 3,5 \\
\hline Zeyhera montana Mart. & 9 & 5,5 \\
\hline Tabebuia impetiginosa (Mart. ex DC.) Standl. & 4 & 3,5 \\
\hline Protium heptaphyllum (Aubl.) March. & 1 & 3,5 \\
\hline Hymenaea stigonocarpa Mart.ex Hayne & 11 & 7,0 \\
\hline Copaifera langsdorffii Desf. & 9 & 5,0 \\
\hline Dimorphandra gardneriana Tul. & 19 & 6,9 \\
\hline Caryocar coriaceum Wittm. & 3 & 22,0 \\
\hline Combretum mellifluum Eichler & 4 & 3,0 \\
\hline Terminalia brasilienses (Cambess.ex A.St.-Hil.) Eichler & 8 & 8,0 \\
\hline Exellodendron cordatum (Hook.f.) Prance & 1 & 12,0 \\
\hline Diospyros hispida A.DC. & 3 & 3,2 \\
\hline Manihot maracasensis Ule & 26 & 4,0 \\
\hline Bowdichia virgilioides Kunth & 119 & 9,5 \\
\hline Pterodon emarginatus Vogel & 15 & 10,0 \\
\hline Vatairea macrocarpa (Benth.) Ducke & 23 & 8,0 \\
\hline Byrsonima crassifolia (L.) Kunth & 26 & 6,5 \\
\hline Mouriri pusa Gardner ex Gardner & 3 & 3,2 \\
\hline Parkia platycephala Benth. & 43 & 10,0 \\
\hline Stryphnodendron coriaceum Benth. & 33 & 7,0 \\
\hline Plathymenia reticulata Benth. & 35 & 7,0 \\
\hline Psidium hians DC. & 2 & 8,0 \\
\hline Tocoyena formosa (Cham.\& Schltdl.) K. Schum. & 8 & 4,0 \\
\hline Alibertia edulis (Rich.) A.Rich.ex DC. & 1 & 1,8 \\
\hline Agonandra brasilienses Miers. & 1 & 1,8 \\
\hline Qualea parviflora Mart. & 3 & 6,5 \\
\hline Salvertia convallariodora A.St.-Hil. & 11 & 7,0 \\
\hline Total de indivíduos & 476 & \\
\hline
\end{tabular}


CONCEIÇÃO, G. M. da; RUGGIERI, A. C.; SILVA, E. O.; GOMES, E. C.; ROCHE, H. M. V.. Especies vegetales y síndromes de dispersión del área de protección ambiental municipal de Inhamum, Caxias, Maranhão, Brasil. Ambi-Agua, Taubaté, v. 6, n. 2, p. 129-142, 2011. (doi:10.4136/ambi-agua.190)

Tabla 3. Número de individuos (porcentaje) por síndrome de dispersión en cada clase de altura para la sabana, del Área de Protección Ambiental Municipal de Inhamum (Caxias/Maranhão).

\begin{tabular}{l|c|c|c|c}
\hline Altura (h) & Zoocoria n (\%) & Anemocoria n (\%) & Autocoria n (\%) & Total \\
\hline $\mathbf{H} \leq \mathbf{2 , 0}$ & $11(26,2)$ & $17(40,5)$ & $14(33,3)$ & 42 \\
$\mathbf{2 , 0}<\mathbf{h} \leq \mathbf{4 , 0}$ & $60(30,2)$ & $98(49,2)$ & $41(20,6)$ & 199 \\
$\mathbf{H}>\mathbf{4 , 0}$ & $46(19,6)$ & $156(66,4)$ & $33(14,0)$ & 235 \\
\hline \multicolumn{1}{c|}{ Total } & $\mathbf{1 1 7}$ & $\mathbf{2 7 1}$ & $\mathbf{8 8}$ & $\mathbf{4 7 6}$ \\
\hline
\end{tabular}

En algunos trabajos como el de Giehl et al. (2007) y Corrêa et al. (2007), fue encontrada una mayor proporción de individuos zoocóricos en todas las clases de altura. Sin embargo, para Huber (1910) las adaptaciones para la diseminación por el viento (anemocoria) son encontradas en árboles altos. Las dispersiones zoocóricas son observadas en especies con determinada altura, debido al predominio de la fauna.

De hecho, cuanto mayor la clase de altura, mayor la proporción de anemocoria (Corrêa et al., 2007), como consecuencia del aumento de la incidencia de vientos en las capas más altas de la atmósfera. Mientras, a zoocoria, tiende a presentar individuos más bajos, ya que próximo del suelo una mayor diversidad de especies animales consiguen accesar a los frutos.

Considerando el número de individuos en cada área, se observó que la zoocoria fue creciente de la sabana sensu stricto hasta la sabana campo sucio, mientras que la anemocoria decrece (aún siendo predominante). Ya la autocoria, varió un poco de la sabana sensu stricto para la sabana y del bosque para el campo sucio (Figura 5).

Corrêa et al. (2007), obtuvieron resultados semejantes al comparar áreas de sabana con diferentes fisionomias. Para dos áreas, una de campo sucio y otra de bosque, los porcentajes de zoocoria fueron mayores que para la sabana sensu stricto.

Aunque la anemocoria haya sido absoluta, hubo una diferencia significativa entre las tres áreas, en cuanto a la distribución de los síndromes de dispersión, basada en el número de individuos muestreados. La anemocoria predomina en las tres áreas, seguida de la zoocoria y autocoria.

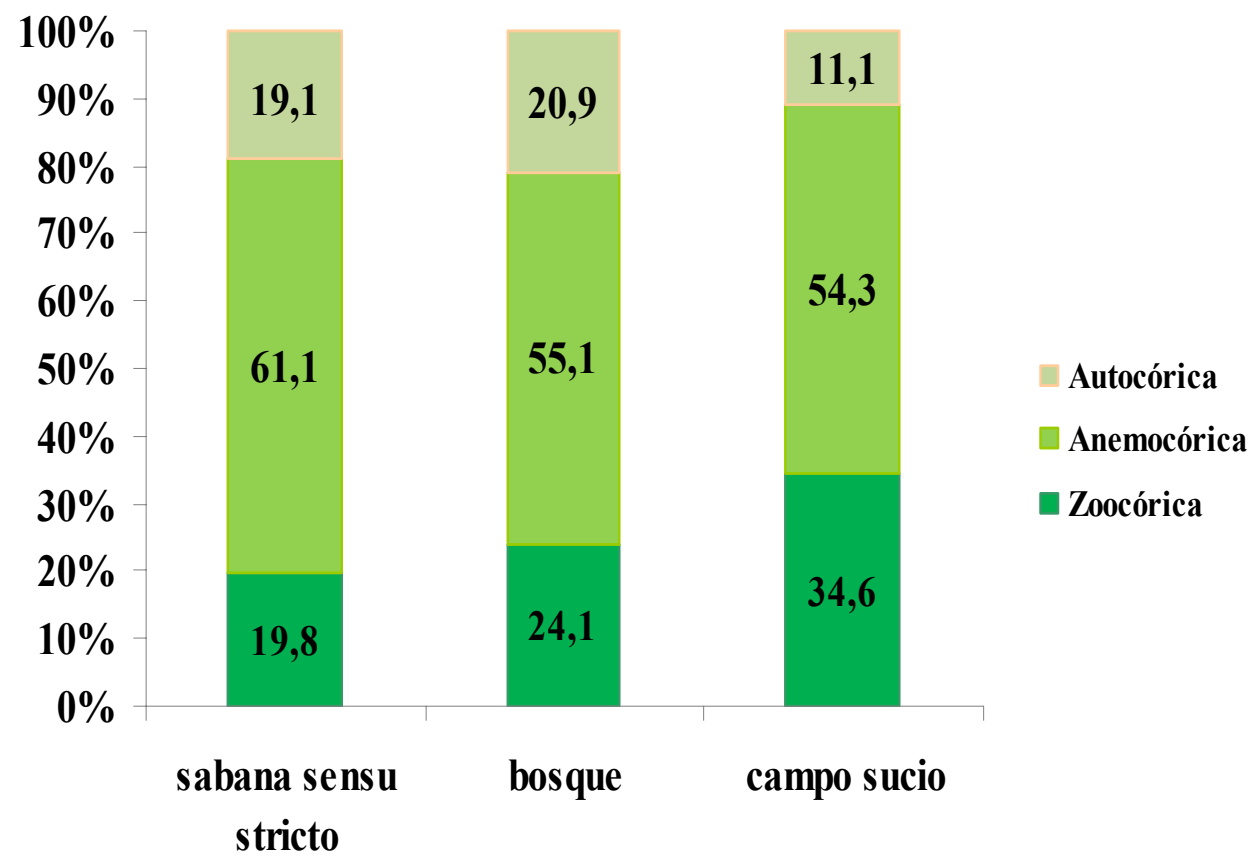

Figura 5. Comparación de la distribución de los síndromes de dispersión por individuos muestreados en las tres áreas de sabana del Área de Protección Ambiental Municipal del Inhamum (Caxias/Maranhão). 
A maior ocorrência de anemocoria nas três áreas pode ser atribuída ao fato de que as áreas más abiertas son más favorables a ese sindrome, como preconizaron Howe e Smallwood (1982). Especies zoocóricas serían más importantes en áreas más cerradas (Morellato y Leitão-Filho, 1992; Talora y Morellato, 2000). Para Gentry (1983), Howe y Smallwood (1982), el mayor o menor porcentaje de especies anemocóricas ha sido relacionado con las variaciones en la precipitación e intensidad de la estación seca. Como resaltaron Marangon (2010), semillas y frutos dispersos por el viento poseen alas o estructuras que disminuyen la velocidad de la caída de los mismos. Sin embargo, el predominio de ese síndrome, además del hecho de ser común en áreas secas, puede estar relacionado también con el grado de perturbación antrópica existente en el Área de Protección Ambiental Municipal del Inhamum. Autores como Griz et al. (2002) y Vicente et al. (2003) argumentan que a medida que aumenta el grado de estacionalidad diminuye la precipitación, los ambientes se tornan más secos y los vectores abióticos, tales como el viento y la gravedad, van ganando importancia.

Varios estudios acerca de los sindromes de dispersión en áreas de sabana muestran la gran importancia de la zoocoria en esos ambientes, seguida de la anemocoria (Giehl et al., 2007; Martins et al., 2007; Seleme y Sartori, 2009; Stefanello et al., 2009; Trindade et al., 2007). En contrapartida, los resultados presentados evidenciaron la importancia de la anemocoria para el mantenimiento del flujo genético de las especies del Área de Protección Ambiental Municipal del Inhamum. En cuanto a la autocoria, los resultados se corroboran con los de otros autores, en cuyos trabajos, el porcentaje es siempre menor. Siendo una estrategia de dispersión raramente encontrada en sabana. Vieira et al. (2002) justificam la rareza, por la pequeña probabilidad de encontrar estas especies, tamaño reducido del número total de estas especies, tamaño del área muestral utilizada, que puede dificultar la presencia de especies autocóricas o entonces la baja eficiencia de estas especies al dispersarse.

La interferencia en ecosistemas como la remoción de la fauna asociada, especialmente por presión de caza, puede llevar a la extinción ecológica y biológica de especies vegetales que dependen de esos animales para dispersarse o mismo para controlar sus poblaciones (Dirzo y Domingues, 1986).

Morellato y Leitão-Filho (1992) consideran que el bajo porcentaje de especies autocóricas parece estar relacionado con el hecho de que estas especies dependen básicamente de la gravedad para su dispersión, no presentando características que relacionen la dispersión de las diásporas con algún agente abiótico o biótico.

\section{CONCLUSIONES}

A anemocoria posee gran importancia para la diseminación de las especies vegetales dentro del área de estudio, siendo esta típica de áreas más secas. Se infiere que el alto grado de perturbación antrópica asociado al clima y a la fisionomía del área de estudio, contribuye significativamente para el predominio de ese síndrome en las especies vegetales. Los datos obtenidos en ese estudio pudieron subsidiar la elaboración de proyectos de manejo y recuperación de áreas degradadas y/o de áreas semejantes, y también refuerzan la importancia de la conservación de estas áreas para el mantenimiento de la diversidad local. Se torna importante resaltar todavía la necesidad de estudios más exactos sobre la dinámica de dispersión de esa área, a fin de confirmar los patrones presentados en este trabajo.

\section{REFERÊNCIAS}

BULLOCK, S. H. Plant reproduction in neotropical dry forests. In: BULLOCK, S. H.; MOONEY, H. A.; MEDINA, E. (Eds.). Seasonally dry tropical forests. London: Cambridge University Press, 1995. p. 277-303. http://dx.doi.org/10.1017/CBO9780511753398.011 
CASTRO, A. A. J. F.; CASTRO, N. M. C. F.; COSTA, J. M.; FARIAS, R. R. S.; MENDES, M. R. A.; ALBINO, R. S.; BARROS, J. S.; OLIVEIRA, M. E. A. Cerrados marginais do Nordeste e ecótonos associados. Revista Brasileira de Biociências, v. 5, supl.1, p. 273-275, 2007.

CAXIAS. Decreto $\mathbf{n}^{\circ}$ 99.274, de 04 de Julho de 2001. Dispõe sobre a Criação da Área de proteção ambiental (APA) Municipal do Inhamum e outras providências. Caxias: Prefeitura Municipal, 2001.

CONCEIÇÃO, G. M.; CASTRO, A. A. F. Fitossociologia de uma área de cerrado marginal, Parque Estadual do Mirador, Mirador, Maranhão. Scientia Plena, v. 5, n. 10, p. 1-16, 2009.

CONCEIÇÃO, G. M.; RUGGIERI, A. C.; GUIMARÃES, E. R. Melastomataceae da Área de Proteção Ambiental Municipal do Inhamum, Caxias, Maranhão. Revista de Biologia e Farmácia, v. 4, n. 2, p. 83-88, 2010.

CORRÊA, C.; CORNETA, C. M.; SCULTORI, C.; VON MATER, S. Síndromes de dispersão em fragmentos de cerrado no município de Itirapina, São Paulo. P. 1-17,2007. Disponível em <http://www2.ibunicamp.br/profs/fsantos/ecocampo/2007/Relatórios /Dispersao.pdf>. Acesso em: 23 de set. 2010.

COSTA, A. A.; ARAÚJO, G. M. Comparação de vegetação arbórea de cerradão e de cerrado na Reserva do Panga, Uberlândia, Minas Gerais. Acta Botanica Brasilica, v. 15, n. 1, p. 63-72, 2000.

DIRZO, R.; DOMINGUEZ, C. A. Seed shadows, seed predation and the advantages of dispersal. In: ESTRADA, A.; FLEMING, T. H. (Eds.). Frugivores and seed dispersal. Dordrech: W. Junk; Berlin: Springer Verlag, 1986. p. 237-249.

GENTRY, A. H. Dispersal ecology and diversity in neotropical forest communities. Sunder. Nature Rev., n. 7, p. 303-314, 1983.

GENTRY, A. H. Diversity and floristic composition of neotropical dry forests. In: BULLOCK, S. H.; MONEY, H. A.; MEDINA, E. (Eds.). Seasonally dry tropical forests. London: Cambridge University Press, 1995. p. 146-190.

http://dx.doi.org/10.1017/CBO9780511753398.007

GIEHL, E. L. H.; ATHAYDE, E. A.; BUDKE, J. C.; GESING, J. P. A.; EINSIGER, S. M.; CANTO-DOROW, T. S. Espectro e distribuição vertical das estratégias de dispersão de diásporos do componente arbóreo em uma floresta estacional no Sul do Brasil. Acta Botanica Brasilica, v. 21, n. 1, p. 137-145, 2007.

http://dx.doi.org/10.1590/S0102-33062007000100013

GRIZ, L. M. S.; MACHADO, I. C. S.; TABARELLI, M. Ecologia de dispersão de sementes: progressos e perspectivas. In: TABARELLI, M.; SILVA, J. M. C. (Orgs.). Diagnóstico da biodiversidade de Pernambuco. Recife: Secretaria de Ciência, Tecnologia e Meio Ambiente; Fundação Joaquim Nabuco; Massangana, 2002. Vol. 2, p. 597-608.

HOWE, H. F.; SMALLWOOD, J. Ecology of seed dispersal. Annual Review Ecology and Systematics, v. 13, p. 201-228, 1982.

http://dx.doi.org/10.1146/annurev.es.13.110182.001221

HUBER, J. Matas e madeiras amazônicas. Boletim Museu Paraense Emílio Goeldi, v. 6, p. 91-225, 1910. 
KLINK, C. A.; MACHADO, R. B. A. A. Conservação do cerrado brasileiro. Megadiversidade, v. 1, n. 1, p. 147-155, 2005.

LIEBSH, D.; ACRA, L. A. Síndromes de dispersão de diásporos de um fragmento de floresta ombrófila mista em Tijucas do Sul, PR. Revista Acadêmica, v. 1, n. 2, p. 167-175, 2007.

LUZ, G. R.; MENINO, G. C. O.; MOTA, G. S.; NUNES, Y. R. F. Síndromes de dispersão de espécies arbustivo-arbóreas em diferentes fitofisionomias no norte de Minas Gerais. In: SIMPÓSIO NACIONAL CERRADO, 9, SIMPÓSIO INTERNACIONAL SAVANAS TROPICAIS, 2. 2008, Brasília. Anais... Brasília: EMBRAPA Cerrados, 2008.

MARANGON, G. P. Dispersão de sementes de uma comunidade arbórea em um remanescente de Mata Atlântica, município de Bonito, PE. Revista Verde, v. 5, n. 5, p. 80-87, 2010.

MARANHÃO. Secretaria de Meio Ambiente e Turismo - SEMATUR. Diagnóstico dos principais problemas ambientais do Maranhão. São Luís: LITHOGRAF, 1991. $194 p$.

MARTINS, F. R.; SANTOS, F. A. M. Técnicas usuais de estimativas da biodiversidade. Revista Holos, p. 236-267, 1999.

MARTINS, M.; ZANZINI, C. S.; SANTIAGO, W. T. V. Síndromes de dispersão em formações florestais do bioma Cerrado no Estado do Tocantins. Revista Brasileira de Biociências, v. 5, supl. 1, p. 807-809, 2007.

MESQUITA, M. R.; CASTRO, A. A. J. F. Florística e fitossociologia de uma área de cerrado marginal (cerrado baixo), Parque Nacional de sete Cidades, Piauí. Public. avulsas conservação de ecossistemas, v.15, p. 1-22, 2007.

MORELlATO, L. P. C.; LEITÃO-FILHO, H. F. Padrões de frutificação e dispersão na Serra do Japi. In: MORELlATO, L. P. C. (Org.). História natural da Serra do Japi: ecologia e preservação de uma área florestal no Sudeste do Brasil. Campinas: Editora da UNICAMP/FAPESP. 1992. p. 112-139.

REBELO, C. G.; FERREIRA, M. E.; ARAÚJO, J. V. G.; STONE, L. F.; SILVA, S. C.; GOMES, M. P. Influência do uso do solo na qualidade da água no bioma Cerrado: um estudo comparativo entre as bacias hidrográficas no Estado de Goiás, Brasil. Revista Ambiente e Água, v. 4, n. 2, p. 172-187, 2009.

REIS, C. S.; CONCEIÇÃO, G. M. Aspectos florísticos de um fragmento de vegetação, localizado no Município de Caxias, Maranhão, Brasil. Scientia Plena, v. 6, n. 20, p. 1$17,2010$.

RIBEIRO, J. F.; WALTER, B. M. T. Fitofisionomias do bioma Cerrado. In: SANO, S. M.; ALMEIDA, S. P. DE (Eds.). Cerrado: ambiente e flora. Planaltina: EMBRAPA, CPAC, 1998. p. 89-166.

SELEME, E. P.; SARTORI, A. L. B. Florística e síndromes de dispersão de espécies arbóreas e arbustivas em Cerradão em Campo Grande, Mato Grosso do Sul. Revista Brasileira de Agroecologia, v. 4, n. 2, p. 3682-3685, 2009. 
STEFANELLO, D.; FERNANDES-BULHÃO, C.; MARTINS, S. V. Síndromes de dispersão de sementes em três trechos de vegetação ciliar (nascente, meio e foz) ao longo do rio Pindaíba, MT. Revista Árvore, v. 33, n. 6, p. 1051-1061, 2009.

http://dx.doi.org/10.1590/S0044-59672010000100018

STEFANELlO, D.; IVANAUSKAS, N. M.; MARTINS, S. V.; KUZ, S. H. Síndromes de dispersão de diásporos das species de trechos de vegetação ciliar do rio das Pacas, Querência - MT. Acta Amazonica, v. 40, n. 1, p. 141-150, 2010.

TALORA, D. C.; MORELlATO, P. C. Fenologia de espécies arbóreas em floresta de planície litorânea do sudeste do Brasil. Revista Brasileira de Botânica, v. 23, p. 13-26, 2000.

TRINDADE, N. P. O.; ZANZINI, A. C. S.; SANTIAGO, W. T. V. Síndromes de dispersão em um gradiente de Cerrado lato sensu no Estado do Tocantins. Revista Brasileira de Biociências, v. 5, supl. 1, p. 897-898, 2007.

VAN DER PIJL, L. V. Principles of dispersal in higher plants. 3. ed. Berlim: SpringerVerlag, 1982.

VICENTE, A.; SANTOS, A. M. M.; TABARELLI, M. Variação no modo de dispersão de espécies lenhosas em um gradiente de precipitação entre floresta seca e úmida no Nordeste do Brasil. In: LEAL, I. R.; TABARELLI, M.; SILVA, J. M. C. (Orgs.). Ecologia e conservação da caatinga. Recife: Editora Universitária da Universidade Federal de Pernambuco, 2003. p. 565-592.

VIEIRA, D. L. M.; AQUINO, F. G.; BRITO, M. A.; FERNANDES-BULHÃO, C.; HENRIQUES, R. P. B. Síndromes de dispersão de espécies arbustivo-arbóreas em cerrado sensu stricto do Brasil Central e savanas amazônicas. Revista Brasileira de Botânica, v. 25, n. 2, p. 215-220, 2002.

YAMAMOTO, L. F.; KINOSHITA, L. S.; MARTINS, F. R. Síndromes de polinização e de dispersão em fragmentos da Floresta Estacional Semidecídua Montana, SP, Brasil. Acta Botânica Brasílica, v. 1, n. 3, p. 553-573, 2007. 